\title{
ENTIRE PROPAGATOR
}

\author{
L. AUTRET AND H. EMAMIRAD
}

(Communicated by Palle E. T. Jorgensen)

\begin{abstract}
We construct an entire solution for an evolutionary Cauchy problem when the initial data is an entire vector of an appropriate operator in a Banach space. We give a sufficient condition for an entire domination. Our results are applied to some partial differential equations including the Dirac system.
\end{abstract}

\section{INTRODUCTION}

In this paper we construct an analytic solution in the whole complex plane for the following initial value evolution problem:

$$
\begin{cases}d u / d z=A(z) u(z) & (z \in \mathbb{C}), \\ u(w)=\phi & (w \in \mathbb{C}) .\end{cases}
$$

Let $A$ be a linear operator on a Banach space $X$, and $D(A)$ its domain. We designate by $\mathscr{E}(A)$ the space of entire vectors defined by

$$
\mathscr{E}(A) \equiv\left\{\phi \in \bigcap_{n=0}^{+\infty} \mathscr{D}\left(A^{n}\right) \mid \sum_{k=0}^{+\infty}\left\|A^{k} \phi\right\| \frac{t^{k}}{k !}<+\infty \quad \forall t \geq 0\right\},
$$

endowed with a Fréchet space topology defined by the calibration $\left\{p_{l}\right\}_{l \in \mathbb{N}}$ where

$$
p_{l}(\phi)=\sum_{k \geq 0}\left\|A^{k} \phi\right\| \frac{l^{k}}{k !} \quad \text { for } \phi \in \mathscr{E}(A) .
$$

In [1], one of the authors showed that if $-A(z)$ is independent of $z$ and generates a strongly continuous holomorphic semigroup, then

$$
\mathscr{E}(A)=Z(A),
$$

where $Z(A)$, is the solution space for $A$ which is the set of all $x \in X$ such that (EP) has a mild solution. Furthermore, if one endows $Z(A)$ with the family of seminorms

$$
\|\phi\|_{n}=\sup _{t \in[0, n]}\|u(t)\|
$$

then $Z(A)$ becomes a Fréchet space (see [dL2]), and the identity (1) holds algebraically and topologically. Our aim in this paper is to investigate a similar

Received by the editors July 20, 1992.

1991 Mathematics Subject Classification. Primary 47D06. 
result in the case where $A(z)$ depends analytically on $z$. To this end we construct an entire propagator under some domination conditions on the family

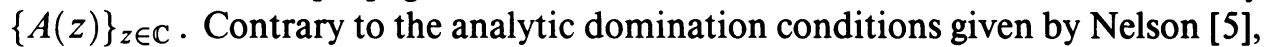
or those of Goodmann and Jorgensen [2], which both have a local character, our domination conditions are more restrictive but ensure sufficient conditions for some linear operator $B$ to get $\mathscr{E}(A) \subseteq \mathscr{E}(A+B)$.

We achieve this paper by applying our results to some evolutionary Cauchy problems of partial differential equations, in particular an important application to the Dirac operator is given.

\section{ENTIRE PROPAGATOR}

All definitions and results concerning analytic functions of several complex variables valued on a locally convex space which are used in the sequel can be found in [3].

Definition 1. A two-parameter family $\{S(z, w)\}_{(z, w) \in \mathbb{C}^{2}}$ of bounded linear operators on a Fréchet space $Y$ is called an entire propagator on $Y$ if the following two conditions are satisfied:

(i) $S(z, z)=I, \quad S(z, \zeta) S(\zeta, w)=S(z, \zeta) \quad(\forall z, \zeta, w \in \mathbb{C})$;

(ii) $(z, w) \longmapsto S(z, w)$ is a strongly holomorphic function on $\mathbb{C}^{2}$.

Let $A$ be a linear closed operator on a Banach space $X$, such that $\mathscr{E}(A) \not \equiv$ $\{0\}$. We assume that the family $\{A(z)\}_{z \in \mathbb{C}}$ satisfies the following assumptions:

(H1) $\mathscr{E}(A) \subseteq \bigcap_{z \in \mathbb{C}} \mathscr{D}(A(z))$;

(H2) For each $\phi \in \mathscr{E}(A), \quad z \longrightarrow A(z) \phi$ is an $\mathscr{E}(A)$-valued entire function;

(H3) There exist two functions $a$ and $b$, bounded on the compact subsets of $\mathbb{C}$ such that

$$
\left\|A^{n} A(z) \phi\right\| \leq a(z)\left\|A^{n+1} \phi\right\|+b(z)\left\|A^{n} \phi\right\|
$$

for all $n \in \mathbb{N}, \phi \in \mathscr{E}(A)$ and $z \in \mathbb{C}$.

The existence theorem can be anounced as follows:

Theorem 1. Suppose that the assumptions $(\mathrm{H} 1)-(\mathrm{H} 3)$ hold. Then there exists an entire propagator $S(z, w)$ on $\mathscr{E}(A)$ such that

(i) $(z, w) \longmapsto S(z, w)$ is holomorphic on $\mathbb{C}^{2}$ for the topology of uniform convergence on the bounded subsets of $\mathscr{E}(A)$;

(ii) $\frac{\partial}{\partial z} S(z, w)=A(z) S(z, w)$; and

(iii) $\frac{\partial}{\partial w} S(z, w)=-S(z, w) A(w)$.

Before starting the proof of this theorem, we give some technical preliminaries.

Lemma 1. Under the same assumptions,

(a) $A \in \mathscr{L}(\mathscr{E}(A))$ and for $R>0, \phi \in \mathscr{E}(A)$ and $k \in \mathbb{N}$, we have

$$
p_{l}\left(A^{k} \phi\right) \leq \frac{k !}{R^{k}} p_{l+l^{\prime}}(\phi) \quad \forall l \geq 0, l^{\prime} \geq R
$$

(b) $p_{t}\left(A^{n} A(z) \phi\right) \leq a(z) p_{t}\left(A^{n+1} \phi\right)+b(z) p_{t}\left(A^{n} \phi\right)$ for all $t \geq 0, \phi \in \mathscr{E}(A), z \in$ $\mathbb{C}$ and $n \in \mathbb{N}$;

(c) $A(z) \in \mathscr{L}(\mathscr{E}(A)) \quad \forall z \in \mathbb{C}$; 
(d) if $z \longmapsto v(z)$ is an $\mathscr{E}(A)$-valued entire function, then $z \longmapsto A(z) v(z)$ is also an $\mathscr{E}(A)$-valued entire function; and

(e) if $(z, w) \longmapsto v(z, w)$ is an $\mathscr{E}(A)$-valued holomorphic function on $\mathbb{C}^{2}$, then $(z, w) \longmapsto \int_{w}^{0} A(\zeta) v(\zeta, w) d \zeta$ is also an $\mathscr{E}(A)$-valued holomorphic function on $\mathbb{C}^{2}$.

Proof. (a) This assumption is a direct consequence of the fact that the series

$$
\sum_{n \geq 0}\left\|A^{k+n} \phi\right\| \frac{z^{n}}{n !}
$$

are convergent and define an entire function when $\phi \in \mathscr{E}(A)$.

(b) (H3) implies that, for any $t \geq 0, \phi \in \mathscr{E}(A), z \in \mathbb{C}$, and $n \in \mathbb{N}$,

$$
\sum_{k=0}^{N}\left\|A^{k} A^{n} A(z) \phi\right\| \frac{t^{k}}{k !} \leq a(z) \sum_{k=0}^{N}\left\|A^{k+n+1} \phi\right\| \frac{t^{k}}{k !}+b(z) \sum_{k=0}^{N}\left\|A^{k+n} \phi\right\| \frac{t^{k}}{k !} .
$$

Letting $N \longrightarrow \infty$ we obtain the desired conclusion.

(c) The continuity of $A(z)$ is a consequence of (b) with $n=0$, of the continuity of $A$ on $\mathscr{E}(A)$ and the fact that for all $t, t^{\prime}$ such that $t \geq t^{\prime}$, $p_{t}(\phi) \geq p_{t^{\prime}}(\phi)$.

(d) For any $t \geq 0$ and $z, \zeta \in \mathbb{C}$

$$
p_{t}\left(\frac{A(z) v(z)-A(\zeta) v(\zeta)}{z-\zeta}-A^{\prime}(\zeta) v(\zeta)-A(\zeta) v^{\prime}(\zeta)\right) \leq A+B+C
$$

where

$$
\begin{aligned}
& A=p_{t}\left(\frac{A(z) v(z)-A(z) v(\zeta)}{z-\zeta}-A(z) v^{\prime}(\zeta)\right) \\
& B=p_{t}\left(\frac{A(z) v(\zeta)-A(\zeta) v(\zeta)}{z-\zeta}-A^{\prime}(\zeta) v(\zeta)\right) \\
& C=p_{t}\left(A(z) v^{\prime}(\zeta)-A(\zeta) v^{\prime}(\zeta)\right)
\end{aligned}
$$

According to $(b)$

$$
A \leq a(z) p_{t}\left(A \frac{v(z)-v(\zeta)}{z-\zeta}-A v^{\prime}(\zeta)\right)+b(z) p_{t}\left(\frac{v(z)-v(\zeta)}{z-\zeta}-v^{\prime}(\zeta)\right) .
$$

Since $a(\cdot)$ and $b(\cdot)$ are bounded on the compact subsets of $\mathbb{C}$, the holomorphy of $z \longmapsto v(z)$ on $\mathscr{E}(A)$ and (a) imply that $A \longmapsto 0$ as $z \longmapsto \zeta$. Cancellation of $B$ and $C$ is a consequence of the strong holomorphy of $A(z)$.

(e) According to (d), for any $w \in \mathbb{C}, \zeta \longmapsto A(\zeta) v(\zeta, w)$ is holomorphic on $\mathbb{C}$. Since $A(z) \in \mathscr{L}(\mathscr{E}(A))$ for any $\psi^{*} \in \mathscr{E}(A)^{*}$, Hartogs Theorem implies that

$$
(\zeta, w) \longmapsto\left\langle v(\zeta, w), A(\zeta)^{*} \psi^{*}\right\rangle
$$

is an entire function on $\mathbb{C}^{2}$. Finally the equivalence between weak and strong holomorphy implies the assertion.

Proof of Theorem 1 . We define inductively

$$
S_{n}(z, w) \phi=\int_{w}^{z} A(\zeta) S_{n-1}(\zeta, w) \phi d \zeta \quad\left(n \in \mathbb{N}^{*}\right)
$$


with $S_{0}(z, w) \phi \equiv \varnothing$. The integral is taken on any contour running from $w$ to $z$. Then we prove by induction that $(z, w) \longmapsto S_{n}(z, w) \phi$ defines an $\mathscr{E}(A)$ valued holomorphic function and that, for all $k \in \mathbb{N}, t \geq 0$, and $z, w \in \mathbb{C}$, there exists $r>0$ such that

$$
p_{t}\left(A^{k} S_{n}(z, w) \phi\right) \leq \frac{(\alpha(r)|z-w|)^{n}}{n !}\left[\sum_{l=0}^{n} C_{n}^{l} p_{t}\left(A^{k+l} \phi\right)\right] .
$$

The number $r$ is such that the contour going from $w$ to $z$ is included in the closed ball $B(0, r)$ and $\alpha(r)=\sup \left(\sup _{z \in B(0, r)} a(z), \sup _{z \in B(0, r)} b(z)\right)$. In fact by Lemma $1(b)$ and by induction we have

$$
\begin{aligned}
& p_{t}\left(A^{k} S_{n+1}(z, w) \phi\right) \\
& \leq \int_{0}^{z-w}\left[a(\zeta+w) p_{t}\left(A^{k+1} S_{n}(\zeta+w, w) \phi\right)\right. \\
& \left.\quad+b(\zeta+w) p_{t}\left(A^{k} S_{n}(\zeta+w, w) \phi\right)\right] d|\zeta| \\
& \quad \leq \alpha(r)^{n+1} \int_{0}^{z-w} \frac{|\zeta|^{n}}{n !}\left[\sum_{l=0}^{n} C_{n}^{l} p_{t}\left(A^{k+l+1} \phi\right)+\sum_{l=0}^{n} C_{n}^{l} p_{t}\left(A^{k+l} \phi\right)\right] d|\zeta|,
\end{aligned}
$$

which proves (2). So assertion (a) of the Lemma 1 together with (2) imply

$$
\begin{aligned}
p_{t}\left(S_{n}(z, w) \phi\right) & \leq \frac{(\alpha(r)|z-w|)^{n}}{n !}\left[\sum_{l=0}^{n} C_{n}^{l} \frac{l !}{R^{l}} p_{t+R}(\phi)\right] \\
& \leq \frac{(\alpha(r)|z-w|)^{n}}{R^{n}} e^{R} p_{t+R}(\phi) .
\end{aligned}
$$

Thus the series

$$
S(z, w)=\sum_{n \geq 0} S_{n}(z, w) \phi
$$

converges uniformly on the compact subsets of $\mathbb{C}^{2}$, and $(z, w) \longmapsto S(z, w) \phi$ is analytic on $\mathbb{C}^{2}$ for all $\phi \in \mathscr{E}(A)$. From relation (3) we get

$$
p_{t}(S(z, w) \phi) \leq \frac{R e^{R}}{R-\alpha(r)|z-w|} p_{t+R}(\phi) .
$$

Consequently, the linear operators $S(z, w)$ are bounded on $\mathscr{E}(A)$ and the families $\{S(z, w) \phi: z \in K\}$ and $\{S(z, w) \phi: w \in K\}$, where $K$ is compact, are equicontinuous. Thus, by virtue of the strong holomorphy of $z \longmapsto S(z, w)$ on $\mathbb{C}$, the partial derivatives $\frac{\partial}{\partial z} S(z, w)$ and $\frac{\partial}{\partial w} S(z, w)$ exist for the uniform topology on the bounded sets of $\mathscr{E}(A)$. Since $\mathscr{E}(A)$ is a Fréchet space, $\mathscr{L}(\mathscr{E}(A))$ equipped with the uniform topology is a complete locally convex space and Hartogs Theorem implies that $S(z, w)$ is analytic for the uniform topology of $\mathscr{L}(\mathscr{E}(A))$. It is easy to verify from (4) that

$$
S(z, w) \phi=\phi+\int_{w}^{z} A(\zeta) S(\zeta, w) \phi d \zeta
$$

Hence $S(z, z)=I$. Property (ii) of Definition 1 together with (ii) of Theorem 1 come from this equality. To prove (iii), we define recursively 
$T_{0}(z, w) \phi \equiv \phi$ and

$$
T_{n}(z, w) \phi=\int_{w}^{z} T_{n-1}(z, \zeta) A(\zeta) \phi d \zeta .
$$

As before we can prove by induction that

$$
p_{t}\left(T_{n}(z, w) \phi\right) \leq \frac{(\alpha(r)|z-w|)^{n}}{n !}\left[\sum_{l=0}^{n} C_{n}^{l} p_{t}\left(A^{l} \phi\right)\right] \quad \forall \phi \in \mathscr{E}(A)
$$

and obtain the convergence of the series

$$
T(z, w)=\sum_{n \geq 0} T_{n}(z, w)
$$

which is holomorphic on $\mathbb{C}^{2}$ for the uniform topology of $\mathscr{L}(\mathscr{E}(A))$. This gives

$$
T(z, w) \phi=\phi+\int_{w}^{z} T(z, \zeta) A(\zeta) \phi d \zeta
$$

for all $\phi \in \mathscr{E}(A)$. So, we have $T(w, w)=I$ and $\frac{\partial}{\partial w} T(z, w)=-T(z, w) \times$ $A(w)$.

Let $z$ and $w$ be complex numbers. The holomorphy of the function $\zeta \longrightarrow$ $T(z, \zeta) S(\zeta, w)$ implies that

$$
\frac{\partial}{\partial \zeta} T(z, \zeta) S(\zeta, w)=-T(z, \zeta) A(\zeta) S(\zeta, w)+T(z, \zeta) A(\zeta) S(\zeta, w)=0
$$

which shows that $T(z, \zeta) S(\zeta, w)$ is independent of $\zeta$. By taking succesively $\zeta=z$ and $\zeta=w$, we have $T(z, w)=S(z, w)$.

The uniqueness of the entire propagator will be a consequence of the following theorem.

Theorem 2. Suppose that the assumptions (H1)-(H3) hold. Then for all $w \in \mathbb{C}$ and all $\phi \in \mathscr{E}(A)$, (EP) has a unique solution $u$ given by $u(z)=S(z, w) \phi$ where $S(z, w)$ is the propagator given by Theorem 1 .

Proof. From (ii) in Theorem 1, it follows that $u$ defined by $u(z)=S(z, w) \phi$ is a solution of (EP). To prove the uniqueness, let $v$ be another solution of (EP). Since $\{S(z, w)\}$ is analytic in $\mathbb{C} \times \mathbb{C}$ for the uniform topology of $\mathscr{L}(\mathscr{E}(A))$, the function $\zeta \longrightarrow S(z, \zeta) v(\zeta)$ is entire and

$$
\frac{\partial}{\partial \zeta} S(z, \zeta) v(\zeta)=-S(z, \zeta) A(\zeta) v(\zeta)+S(z, \zeta) A(\zeta) v(\zeta)=0
$$

Consequently, $S(z, \zeta) v(\zeta)$ is constant in $\zeta$. Letting $\zeta=z$ and $\zeta=w$ we find $S(z, w) \phi=v(z)$. This uniquness result implies the uniqueness of the propagator $S(z, w)$ in the sense of Definition 1 .

Corollary 1. Suppose that the assumptions (H1)-(H3) hold. Then the Cauchy problem for $(E P)$ is well posed in $\mathscr{E}(A)$. More precisely, for any initial data in $\mathscr{E}(A)$, for any continuous seminorm $p_{t}$, and any compact subset $K$ of $\mathbb{C}^{2}$, there exists $M \in \mathbb{R}^{+}$and a continuous seminorm $p_{t^{\prime}}$ such that

$$
p_{t}(u(z, w)) \leq M p_{t^{\prime}}(\phi) \quad \forall(z, w) \in K .
$$

Proof. The proof is a direct consequence of Theorem 1 and of the local equicontinuity of the entire propagator on the compact subsets of $\mathbb{C}^{2}$. 
Remark 1. If there exists a complex number $\lambda,|\lambda| \leq 1$, such that $A A(z)=$ $\lambda A(z) A$ and

$$
\|A(z) \phi\| \leq a(z)\|A \phi\|+b(z)\|\phi\|
$$

for all $z \in \mathbb{C}$ and all $\phi \in \mathscr{E}(A)$ with the assumptions on $a$ and $b$ as in (H3), then the assumptions $(\mathrm{H} 1)-(\mathrm{H} 3)$ are satisfied. In particular, this is the case when $A$ and $A(z)$ commute.

Remark 2. It is not hard to see that we can improve assertion (H3) by replacing it with: there exist $m$ functions $\left\{a_{j}: j=1, \ldots, m\right\}$, bounded on compact subsets of $\mathbb{C}$, such that, for all $n \in \mathbb{N}, \phi \in \mathscr{E}(A)$, and $z \in \mathbb{C}$,

$$
\left\|A^{n} A(z) \phi\right\| \leq \sum_{j=1}^{m} a_{j}(z)\left\|A^{n+j} \phi\right\|,
$$

and get the same result as Theorem 1. Suppose now that $A(z)$ is independent of $z$ and $A(z)=A+B$; then Theorem 1 gives a theorem of perburbation for entire vectors. The following corollary makes this notion more precise.

Corollary 2. Let $A$ be a closed linear operator on $X$ such that $\mathscr{E}(A) \not \equiv\{0\}$. Suppose $B$ is a linear operator for which there exist two constants $a \geq 0$ and $b \geq 0$ such that

$$
\left\|A^{n} B \phi\right\| \leq a\left\|A^{n+1} \phi\right\|+b\left\|A^{n} \phi\right\| \quad \forall n \in \mathbb{N}, \phi \in \mathscr{E}(A) .
$$

Then $e^{z(A+B)} \phi$ exists on $\mathscr{E}(A)$ and $\mathscr{E}(A) \subseteq \mathscr{E}(A+B)$.

Proof. Take in Theorem 1, w=0 and $A(z)=A+B$. This implies that the series

is convergent in $\mathscr{E}(A)$.

$$
\sum_{n \geq 0}(A+B)^{n} \phi \frac{z^{n}}{n !}
$$

\section{EXAMPLES}

1. A parabolic partial differential equation. Let $\Omega$ be a bounded domain with smooth boundary $\partial \Omega$ in $\mathbb{R}^{n}$. In this section, we consider on $L^{p}(\Omega)$ $(1<p<\infty)$ the following linear evolution problem:

$$
\begin{cases}\frac{\partial u(z, x)}{\partial z}=A(z, D) u(z, x) & (x \in \Omega, z \in \mathbb{C}), \\ u(z, x)=0 & (x \in \partial \Omega, z \in \mathbb{C}), \\ u(0, x)=\phi(x) & (x \in \Omega),\end{cases}
$$

with $A(z, D)=\sum_{|\alpha| \leq 2 m} a_{\alpha}(z) D^{\alpha}$.

We define $|\alpha|=\sum_{j=1}^{n} \alpha_{j}, x^{\alpha}=x_{1}^{\alpha_{1}} \cdots x_{n}^{\alpha_{n}}$ and $D^{\alpha}=D_{1}^{\alpha_{1}} \cdots D_{n}^{\alpha_{n}}$ where $D_{j}=\partial / \partial x_{j}$.

For all $\alpha$ such that $|\alpha| \leq 2 m$, we suppose the function $z \longrightarrow a_{\alpha}(z)$ is entire. The operator $A(z)$ is defined by $\mathscr{D}(A(z))=W^{2 m, p}(\Omega) \cap W_{0}^{m, p}(\Omega)$ and $A(z) f=A(z, D) f$ for all $f \in \mathscr{D}(A(z))$. Let $A$ be a strongly elliptic operator of order $2 m$ defined on $L^{p}(\Omega)$ by $\mathscr{D}(A)=W^{2 m, p}(\Omega) \cap W_{0}^{m, p}(\Omega)$. For such operators, we have the following estimate (see, e.g., [6]).

There exists a constant $C \geq 0$ such that

$$
\|u\|_{2 m, p} \leq C\left(\|A u\|_{p}+\|u\|_{p}\right) \quad \forall u \in W^{2 m, p}(\Omega) \cap W_{0}^{m, p}(\Omega) .
$$


Since $A$ generates a holomorphic strongly continuous semigroup and $\mathscr{E}(A)$ is dense in $L^{p}(\Omega)$ (see [4]),

$$
\|A(z) \phi\|_{p} \leq \sum_{|\alpha| \leq 2 m} \mid a_{\alpha}(z)\|\| D^{\alpha} \phi \|_{p} \leq a(z)\left[\|A \phi\|_{p}+\|\phi\|_{p}\right]
$$

and Remark 1 imply that (P1) admits a unique solution $u \in \mathscr{H}(\mathbb{C}, \mathscr{E}(A))$.

2. An integro-differential equation. Let $\Delta$ be the Laplacian operator on $L^{2}\left(\mathbb{R}^{m}\right)$ and $A(z)$, the operator defined for all $z \in \mathbb{C}$ on $L^{2}\left(\mathbb{R}^{m}\right)$ by $A(z) u=-\Delta u+$ $K(z) * u$ and $\mathscr{D}(A(z))=\mathscr{D}(-\Delta)$. We assume that $z \longmapsto K(z)$ is a $L^{1}\left(\mathbb{R}^{m}\right)$ valued entire function.

The space $\mathscr{E}(-\Delta)$ is dense (see [4]), and we consider in this space the abstract Cauchy problem

$$
\left\{\begin{array}{l}
d u(z) / d z=A(z) u(z) \\
u(0)=\varnothing
\end{array}\right.
$$

Let $\|\cdot\|_{2}$ and $\|\cdot\|_{1}$ be the respective norms of $L^{2}\left(\mathbb{R}^{m}\right)$ and of $L^{1}\left(\mathbb{R}^{m}\right)$. If $\phi \in \mathscr{E}(-\Delta)$, then

$$
\|K(z) * \phi\|_{2} \leq\|K(z)\|_{1}\|\phi\|_{2} .
$$

By virtue of the commutation of $\Delta$ and $A(z)$, we are in the framework of Remark 1 and

$$
p_{t}\left(\left[\left(\frac{K(z)-K\left(z^{\prime}\right)}{z-z^{\prime}}-K^{\prime}\left(z^{\prime}\right)\right) * \phi\right]\right) \leq\left\|\frac{K(z)-K\left(z^{\prime}\right)}{z-z^{\prime}}-K^{\prime}\left(z^{\prime}\right)\right\|_{1} p_{t}(\phi)
$$

for all $t \geq 0$ and $n \in \mathbb{N}$; thus, Theorem 1 can be applied. Hence (P2) admits a unique solution $u \in \mathscr{H}(\mathbb{C}, \mathscr{E}(-\Delta))$.

3. Dirac operator. The Dirac operator for a free electron is defined by $\mathbb{D}_{\hbar}=$ $\mathbb{A}+\frac{c^{2} \mu}{i \hbar} \mathbb{J}$, where

$$
\mathbb{A}=c\left(\begin{array}{cc}
0 & A \\
A & 0
\end{array}\right), \quad \text { with } A=\left(\begin{array}{cc}
\frac{\partial}{\partial x_{3}} & \frac{\partial}{\partial x_{1}}-i \frac{\partial}{\partial x_{2}} \\
\frac{\partial}{\partial x_{1}}+i \frac{\partial}{\partial x_{2}} & -\frac{\partial}{\partial x_{3}}
\end{array}\right),
$$

and

$$
\mathbb{J}=\left(\begin{array}{cc}
I & 0 \\
0 & -I
\end{array}\right), \quad \text { with } I=\left(\begin{array}{ll}
1 & 0 \\
0 & 1
\end{array}\right) .
$$

Here $\mu$ is the mass of the electron, $c$ is the speed of light, and $\hbar$ is Planck's constant. $i \mathbb{A}$ is selfadjoint on the Hilbert space $H=L^{2}\left[\mathbb{R}^{3}, \mathscr{L}\left(\mathbb{C}^{4}\right)\right]$, so that $\mathscr{E}(\mathbb{A})$ is dense in $H$. Physically, one is interested on the behavior of $u_{\hbar}(t, x)$, the solution of $\frac{d}{d t} u=\mathbb{D}_{\hbar} u$, as $\hbar \rightarrow 0$. Instead of this, we replace $\frac{c^{2} \mu}{i \hbar}$ by $z$ and look to the holomorphy of $z \longmapsto u(z)$, where $u(z)$ is the solution of $\frac{d}{d z} u=\mathbb{D}_{z} u=(\mathbb{A}+z \mathbb{J}) u$, on a neighborhood of imaginary axes. In spite of the fact that $\mathbb{A}$ and $\mathbb{J}$ are not commutative, however, we are in the scope of the Remark 1 and we have $\left\|\mathbb{D}_{z} u\right\| \leq\|\mathbb{A} u\|+|z|\|u\|$. Thus, Theorem 1 is applicable and $u(z) \in \mathscr{H}(\mathbb{C}, \mathscr{E}(\mathbb{A}))$ whenever $u(0) \in \mathscr{E}(\mathbb{A})$. 


\section{REFERENCES}

1. L. Autret, Entire vectors and time reversible Cauchy problems, Semigroup Forum 46 (1993), 347-351.

2. F. Goodman and P. E. T. Jorgensen, Lie algebras of unbounded derivations, J. Funct. Anal. 52 (1983), 369-384.

3. M. Herve, Analyticity in infinite dimensional spaces, Walter de Gruyter, Berlin and New York, 1989.

4. R. deLaubenfels, Functional calculus, existence families and the abstract Cauchy problem, Lecture Notes in Math., Springer-Verlag, New York (to appear).

5. E. Nelson, Analytic vectors, Ann. of Math. (2) 70 (1959), 572-615.

6. A. Pazy, Semigroups of linear operators and applications to partial differential equations, Springer, Berlin and New York, 1983.

Département de Mathématiques, Université de Poitiers, 86022 Poitiers cedex, France 